\title{
Mixed convection heat transfer in a lid driven cavity with wavy bottom surface
}

\author{
Litan Kumar Saha ${ }^{1,}$, Monotos Chandra Somadder ${ }^{1}$, K. M. Salah Uddin ${ }^{2}$ \\ ${ }^{1}$ Department of Mathematics, University of Dhaka, Dhaka, Bangladesh \\ ${ }^{2}$ Department of Management Information Systems, University of Dhaka, Dhaka, Bangladesh
}

\section{Email address:}

lksaha@univdhaka.edu (L. K. Saha), salahuddin@du.ac.bd (K. M. Salah Uddin)

\section{To cite this article:}

Litan Kumar Saha, Monotos Chandra Somadder, K. M. Salah Uddin. Mixed Convection Heat Transfer in a Lid Driven Cavity with Wavy Bottom Surface. American Journal of Applied Mathematics. Vol. 1, No. 5, 2013, pp. 92-101. doi: 10.11648/j.ajam.20130105.11

\begin{abstract}
The present numerical study is devoted to investigate the mixed convection flow and heat transfer in a lid-driven cavity with wavy bottom surface. The cavity upper wall is moving with a uniform velocity by unity and the other walls are no slip. The cavity vertical walls are insulated while the upper surface is maintained at a uniform temperature higher than the wavy bottom surface. The physical problem is represented mathematically by a set of governing equations and the developed mathematical model is solved by employing Galerkin weighted residual method of finite element formulation. The wide ranges of governing parameters, i. e., the Reynolds number (Re), the Grshof number ( $\mathrm{Gr}$ ) and the number of undulations $(\lambda)$ on the flow structure and heat transfer characteristics are investigated in detail. It is found that these parameters have significant effect on the flow fields; temperature distributions and heat transfer in the cavity. Furthermore, the trend of skin friction and Nusselt number for different values of the aforementioned parameters are presented in this investigation.
\end{abstract}

Keywords: Mixed Convection, Wavy Surface, Lid Driven Cavity, Heat Transfer, Numerical Study

\section{Introduction}

Combined forced and free convection flow in lid-driven cavities or enclosures occurs because of two competing mechanisms. The first is due to shear flow caused by the movement of one of the walls of the cavity while the second is due to buoyancy flow produced by thermal non-homogeneity of the cavity boundary. The fundamental problem of combined forced and natural convection heat transfer in a cavity has received considerable attention from researchers. This problem is often encountered in many engineering and industrial applications, such as solar ponds [1], cooling of electronic devices, heat exchangers, materials processing, crystal growth, float glass production [2], dynamics of lakes [3], metal coating and casting, and among others. Prasad and Koseff [4] have investigated combined forced and natural convection heat transfer in a deep lid-driven cavity flow problem. Investigation has been carried out for various values of Richardson number between 0.1 and 1000 and they found that the heat transfer within the cavity is independent of the Richardson number.

Augmentation of flow and heat transfer in geometries with roughen surfaces is a topic of fundamental importance. This interest stems from its significance in many engineering and industrial applications such as cooling of electrical and nuclear components. Sometimes surfaces are intentionally roughened to enhance heat transfer. Das and Mahmud [5] conducted a numerical investigation of natural convection in an enclosure consisting of two isothermal horizontal wavy walls and two adiabatic vertical straight walls. Adjlout et al. [6] has studied laminar natural convection in an inclined cavity with a heated undulated wall, i.e., smooth wave-like pattern. Their results concluded that the hot wall undulation affects the flow and heat transfer rate in the enclosure in which the local Nusselt number distribution results in a decrease of heat transfer rate as compared with the square enclosure. Moreover, Kumar [7] conducted a study of flow and thermal field inside a vertical wavy enclosure filled with a porous media. The author has illustrated that the surface temperature was very sensitive to the drifts in the surface undulations, phase of the wavy surface and the number of waves considered.

The flow within a lid driven cavity consisting of hot upper wall and cool sinusoidal bottom wall is an important circumstance encountered quite frequently in practice. In all the applications having this kind of situation, heat 
transfer occurs due to the temperature difference across the fluid layer, one horizontal solid surface being at a temperature higher than the other. If the upper plate is the hot surface, then the lower surface has heavier fluid and by virtue of buoyancy, the fluid would not come to the lower plate. Because in this case the heat transfer mode is restricted to only conduction. Convection is the heat transfer mechanism affected by the flow of fluids. The amount of energy and matter are conveyed by the fluid can be predicted through the convective heat transfer. The convective heat transfer splits into two branches; the natural convection and the forced convection. Forced convection regards the heat transport by induced fluid motion which is forced to happen. This induced flow needs consistent mechanical power. However, natural convection differs from the forced convection through the fluid flow driving force, which happens naturally. The flows are driven by the buoyancy effect due to the presence of density gradient and gravitational field. As the temperature distribution in the natural convection depends on the intensity of the fluid currents which is dependent on the temperature potential itself, the qualitative and quantitative analysis of natural convection heat transfer is very difficult. Numerical investigation instead of theoretical analysis is more needed in this field.

The problem of mixed convective heat transfer in a cavity subjected to sinusoidal cool boundary at the bottom surface has not yet been dealt much in the literature. Khanafer et al. [8] carried out a numerical study on natural convection heat transfer inside a porous cavity with a sinusoidal vertical wavy wall. Their results showed that the amplitude of the wavy surface and the number of undulations affect heat transfer characteristics inside the cavity. Al-Amiri et al. [9] performed the effects of mixed convection heat transfer in lid-driven cavity with sinusoidal wavy bottom surface. They observed that the average Nusselt number increases with the increasing values of Reynolds number and amplitude of the wavy surface.

The objective of the present study is to examine the momentum and energy transport processes in a lid-driven cavity with wavy bottom surface. The cavity is sustained under a vertical temperature gradient by subjecting the upper lid to a relatively higher temperature than the wavy bottom wall. The results are shown in terms of parametric presentations of streamlines and isotherms for various considered pertinent dimensionless parameters. These dimensionless groups include the Reynolds number, Grashof number, the wavy surface amplitude, and number of undulations offered by the wavy bottom surface. Finally, the implications of the above dimensionless parameters are also depicted on the dimensionless local heat flux.

\section{Mathematical Formulation}

A two dimensional square cavity of height and length $L$ with sinusoidal bottom surface, as shown in Fig. 1, is considered. The upper wall of the cavity is allowed to move in its own plane at a constant speed $U_{0}$ and the other walls have no-slip condition. The cavity bottom wall is kept at a low temperature, $T c$, and the upper wall is kept at a high temperature, $T h$. The left and right walls are considered to be adiabatic. Such an imposed temperature condition will render a thermal stratification in the cavity. The thermo-physical properties of the fluid are assumed to be constant, except the density in the buoyancy term. The density varies linearly with temperature as $\left(\rho-\rho_{c}\right)=g \beta\left(T-T_{c}\right)$, where $g$ is the acceleration due to gravity and $\beta$ is the coefficient of thermal expansion, $\rho$ is the fluid density. In general, the cavity fluid is assumed to be Newtonian and incompressible, unsteady and laminar flow. It is further assumed that viscous dissipation is neglected in the study.

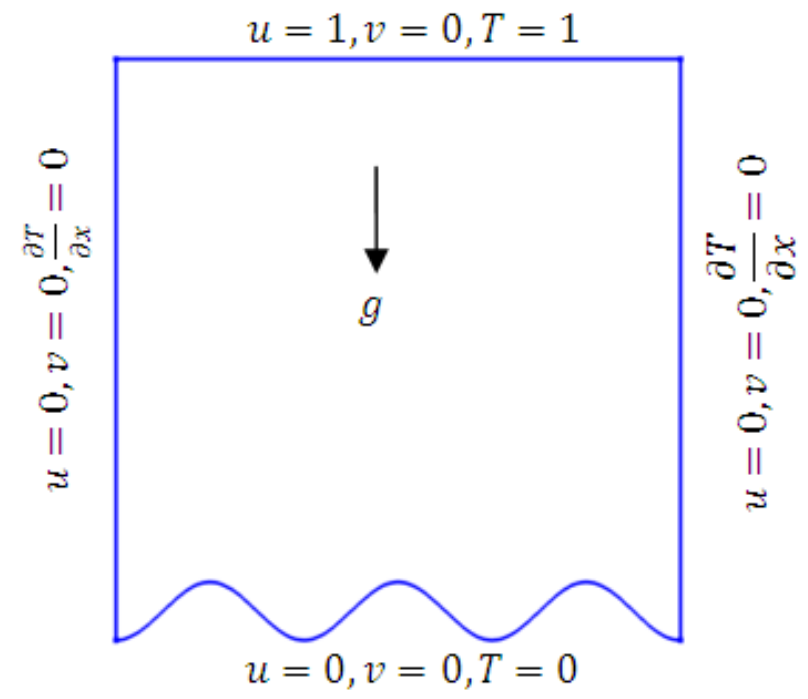

Fig 1. Cavity geometry and boundary condition

With abovementioned assumptions, the governing equations for conservations of mass, momentum and energy can be written as

Mass conservation equation:

$$
\frac{\partial u}{\partial x}+\frac{\partial v}{\partial y}=0
$$

Momentum conservation equation:

$$
\begin{gathered}
u \frac{\partial u}{\partial x}+v \frac{\partial u}{\partial y}=-\frac{1}{\rho} \frac{\partial p}{\partial x}+v\left(\frac{\partial^{2} u}{\partial x^{2}}+\frac{\partial^{2} u}{\partial y^{2}}\right) \\
u \frac{\partial v}{\partial x}+v \frac{\partial v}{\partial y}=-\frac{1}{\rho} \frac{\partial p}{\partial y}+v\left(\frac{\partial^{2} v}{\partial x^{2}}+\frac{\partial^{2} v}{\partial y^{2}}\right)+g \beta\left(T-T_{c}\right)
\end{gathered}
$$

Energy conservation equation:

$$
u \frac{\partial T}{\partial x}+v \frac{\partial T}{\partial y}=\alpha\left(\frac{\partial^{2} T}{\partial x^{2}}+\frac{\partial^{2} T}{\partial y^{2}}\right)
$$


where $u$ and $v$ the fluid velocity components in the $x$ - and $y$ directions, $p$ the pressure, $x, y$ the coordinate directions, $T$ the temperature, $\beta$ the volumetric thermal expansion coefficient, $\rho$ the density of the fluid and $\alpha$ the thermal diffusivity.

The governing equations (1)-(4) are non-dimensionalized using the following dimensionless variables:

$$
X=\frac{x}{L}, Y=\frac{y}{L}, U=\frac{u}{U_{0}}, V=\frac{v}{U_{0}}, P=\frac{p}{\rho U_{0}^{2}}, \theta=\frac{T-T_{c}}{T_{h}-T_{c}}
$$

Introducing the above non-dimensional scales into the governing equations, we obtain the non-dimensional form of the equations as follows:

$$
\begin{aligned}
& \frac{\partial U}{\partial X}+\frac{\partial U}{\partial Y}=0 \\
& U \frac{\partial U}{\partial X}+V \frac{\partial U}{\partial Y}=-\frac{\partial P}{\partial X}+\frac{1}{\operatorname{Re}}\left(\frac{\partial^{2} U}{\partial X^{2}}+\frac{\partial^{2} U}{\partial Y^{2}}\right) \\
& U \frac{\partial V}{\partial X}+V \frac{\partial V}{\partial Y}=-\frac{\partial P}{\partial Y}+\frac{1}{\operatorname{Re}}\left(\frac{\partial^{2} V}{\partial X^{2}}+\frac{\partial^{2} V}{\partial Y^{2}}\right)+\frac{G r}{\operatorname{Re}^{2}} \theta \\
& U \frac{\partial \theta}{\partial X}+V \frac{\partial \theta}{\partial Y}=\frac{1}{\operatorname{Re} \operatorname{Pr}}\left(\frac{\partial^{2} \theta}{\partial X^{2}}+\frac{\partial^{2} \theta}{\partial Y^{2}}\right)
\end{aligned}
$$

In the above equations $\operatorname{Re}=\frac{U_{0} L}{v}, \quad \operatorname{Pr}=\frac{v}{\alpha}$ and $G r=\frac{g \beta \Delta T L^{3}}{v^{2}}$ are respectively the non-dimensional Reynolds number, Prandtl number and Grashof number. The shape of the bottom wavy surface profile is assumed to mock the following pattern $Y=A(1-\operatorname{Cos}(2 \lambda \pi X))$ where $A$ is the dimensionless amplitude of the wavy surface and $\lambda$ is the number of undulations.

The dimensionless boundary conditions are:

$$
\begin{array}{ll}
U=1, V=0, \theta=1 & \text { for } 0 \leq X \leq 1, Y=1 \\
U=V=0, \theta=0 & \text { for } 0 \leq X \leq 1, Y=A(1-\operatorname{Cos}(2 \lambda \pi X)) \\
U=V=0, \frac{\partial \theta}{\partial X}=0 & \text { for } 0 \leq Y \leq 1, X=0 \& X=1
\end{array}
$$

Since the convective heat transfer coefficient: $h$ and Nusselt number $\mathrm{Nu}$, depend on the temperature gradient at a flat surface, $\left(-\frac{\partial T}{\partial n}\right)$ where $\boldsymbol{n}$ is normal direction to the surface. Therefore, we can obtain the rate of heat flux from each of the walls. The corresponding average Nusselt number at the heated wall is defined as:

$$
N u_{a v}=\frac{1}{S} \int_{0}^{s} N u d x
$$

$S$ is the total chord length of the wavy surface and $s$ is the coordinate along the wavy surface.

\section{Numerical Scheme}

The governing equations along with the boundary conditions are solved through the Galerkin weighted residual finite element method. The formulation of this method and computational procedure is well described by Taylor and Hood [10] and Dechaumphai [11]. The computational domain is discretized employing the uniform mapped mesh grid system. Then the Galerkin weighted residual technique is used to convert the nonlinear governing partial differential equations into a system of integral equations that can be solved numerically. The integration involved in each term of these equations is performed by using Gauss's quadrature method which leads to a set of non-linear algebraic equations. These equations are then modified by imposing boundary equations which is transferred into linear algebraic equations by Newton-Raphson iteration. Finally, these linear equations are solved by applying Triangular Factorization method.

\section{Results and Discussions}

In this section, numerical results are presented for different values of $R e, G r, A, \lambda$. Prandtl number was treated as 0.71 for all cases. The basic features of flow and heat transfer in the lid driven cavity are analyzed with the help of streamlines patterns and isotherms contour. Also, the local Nusselt number, Skin friction, average Nusselt numbers along cooled wavy bottom surface for various undulations are plotted.

Numerical validation: To validate the present numerical code, the results for mixed convection flow in an enclosed cavity with heated upper wall have been compared with those obtained by Iwatsu et al. [12]. The comparison of the results obtained from present code with those of Iwatsu et al. [50] is demonstrated for two different Grashof number, $\mathrm{Gr}=102,106$ at $\mathrm{Re}=103$ in Fig. 2 and Fig. 3 respectively. As seen from these figures the obtained results show very good agreement with the documented study.

In addition, the results acquired by present code also has been validated by comparing the average Nusselt number with that of the results obtained by Iwatsu et al. [12] and Mamun et al. [13], is shown in the Table-1. It is noted that Iwatsu et al. [12] considers a square cavity with heated upper moving lid and Mamun et al. [13] considers a trapezoidal cavity with heated upper moving lid. From the above table we see that the present result have a good agreement with those of Iwatsu et al. [12] and Mamun et al. [13].

From the above discussion, we can say that the present code is valid for laminar mixed convection flow and heat transfer in a lid-driven cavity. 

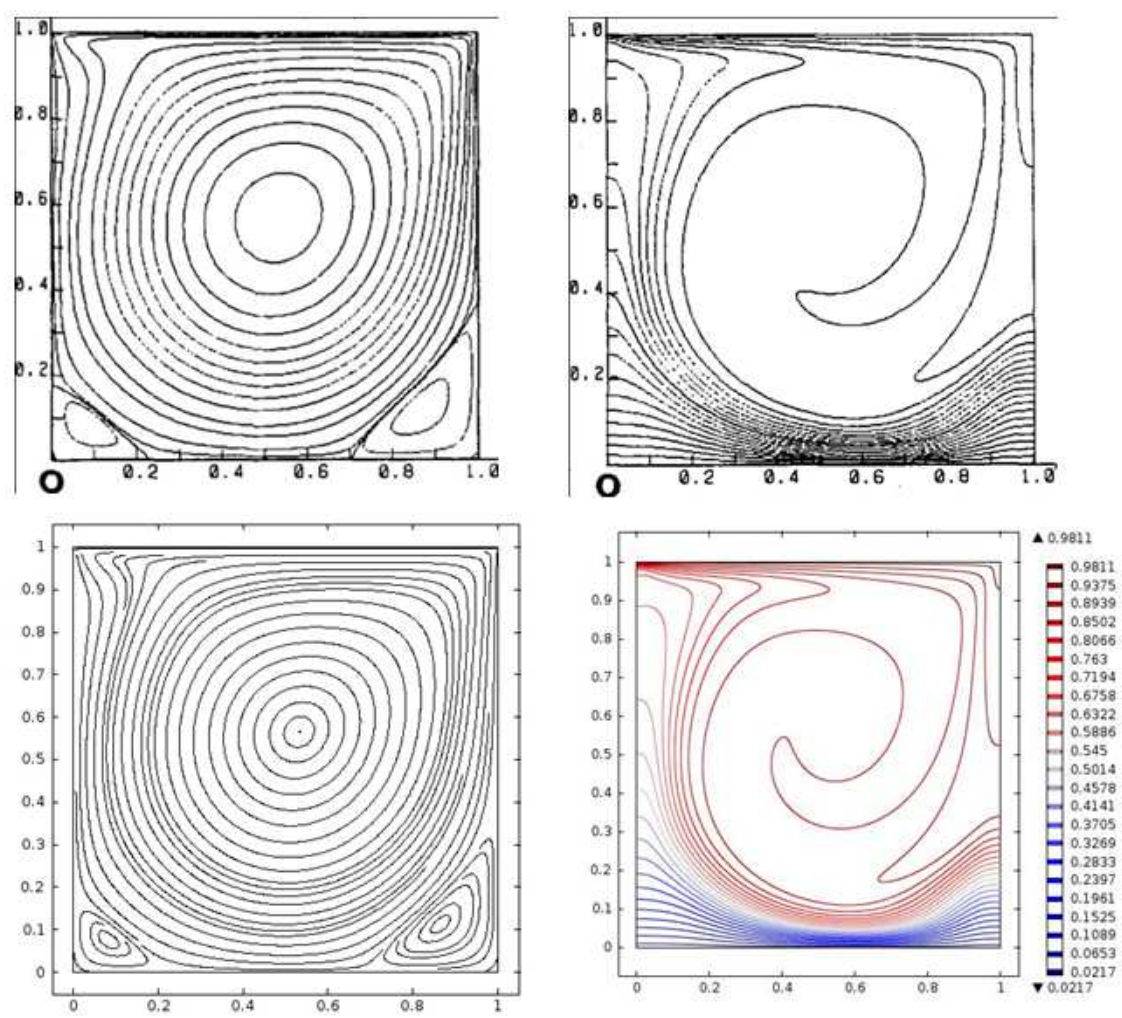

Fig 2. Comparison of streamlines contour (left) and temperature contour (right) obtained by present code (lower) and Iwatsu et al. (upper) at Re $=10^{3}$, $G r=10^{2}$ and $\lambda=0$
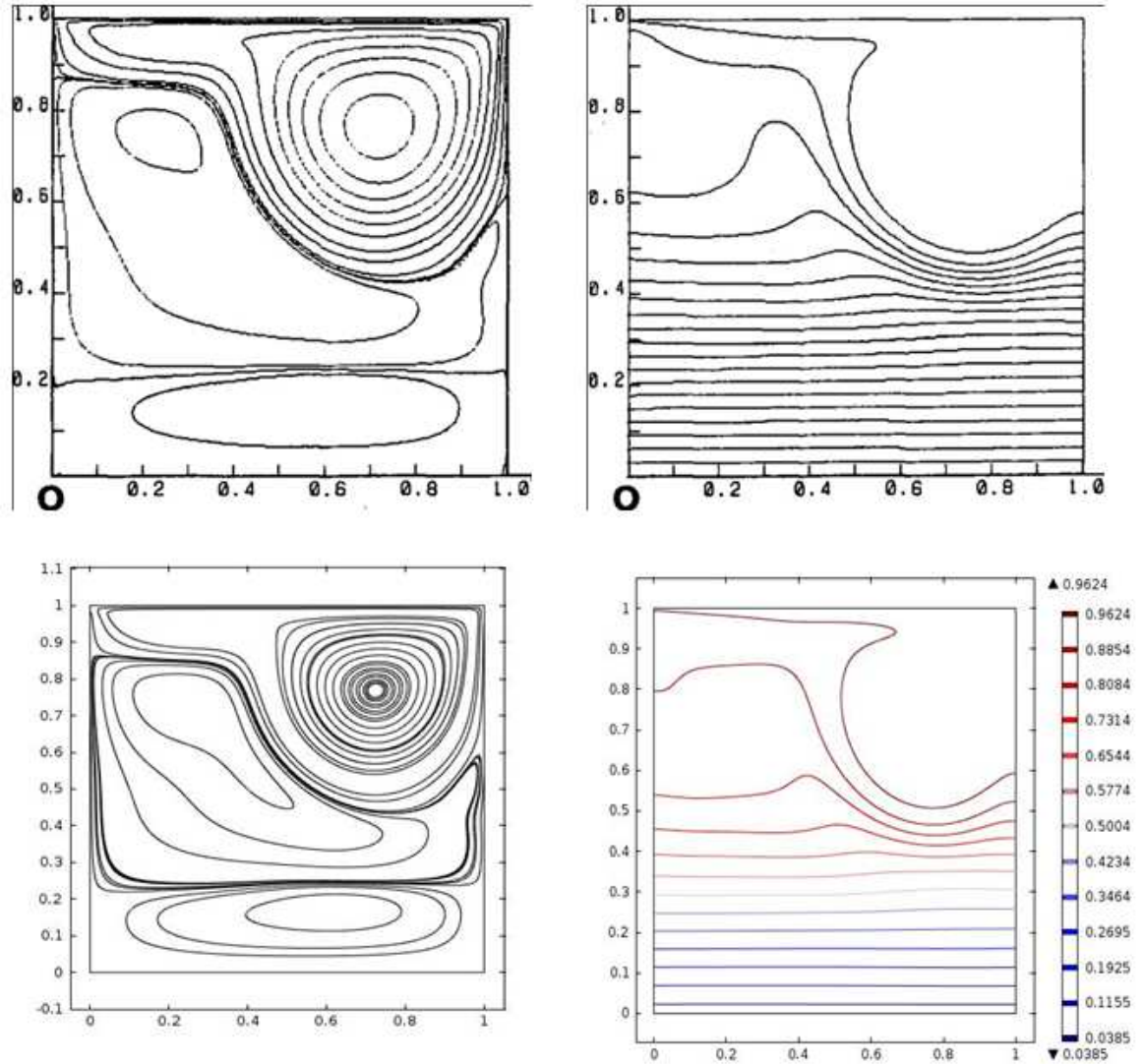

Fig 3. (a) Comparison of stream lines contour (left) and temperature contour (right) between present code (lower) and Iwatsu et al. (upper) at Re=10 ${ }^{3}$, Gr $=10^{6}, \lambda=0$. 
Table 1. Comparison of average Nusselt number with those of Iwatsu et al. and Mamun let.al.

\begin{tabular}{|c|c|c|c|c|c|c|c|c|c|}
\hline \multirow[t]{2}{*}{$\operatorname{Re}$} & \multicolumn{3}{|c|}{$G r=10^{2}$} & \multicolumn{3}{|c|}{$G r=10^{4}$} & \multicolumn{3}{|c|}{$G r=10^{6}$} \\
\hline & Present & Iwatsu & Mamun & Present & Iwatsu & Mamun & Present & Iwatsu & Mamun \\
\hline 100 & 2.01 & 1.94 & & 1.38 & 1.34 & & 1.02 & 1.02 & \\
\hline 400 & 3.97 & 3.84 & 3.97 & 3.72 & 3.62 & 3.75 & 1.18 & 1.22 & 1.18 \\
\hline 1000 & 6.28 & 6.33 & 6.25 & 6.23 & 6.29 & 6.32 & 1.64 & 1.77 & 1.70 \\
\hline
\end{tabular}

(a)

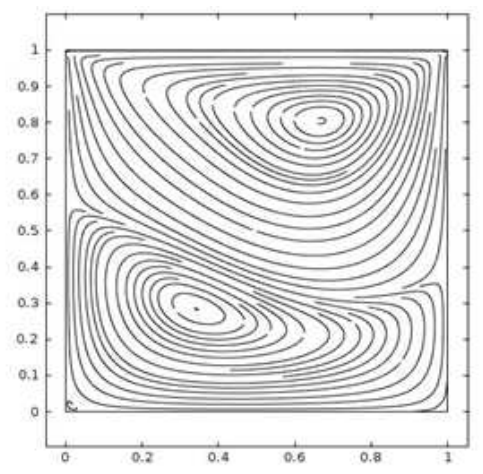

(c)

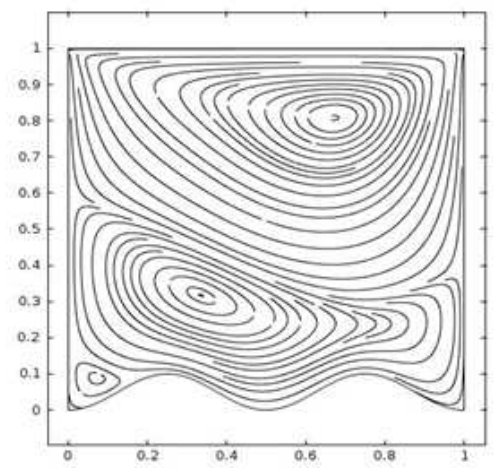

(b)

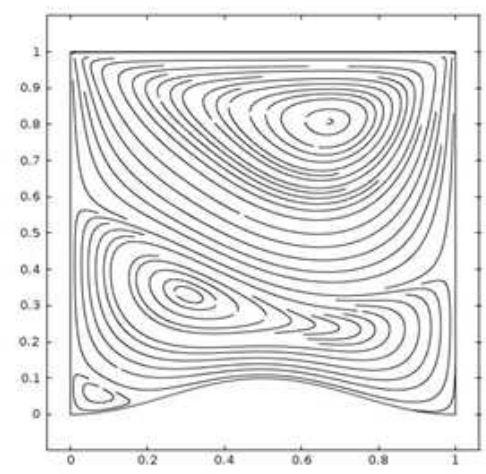

(d)

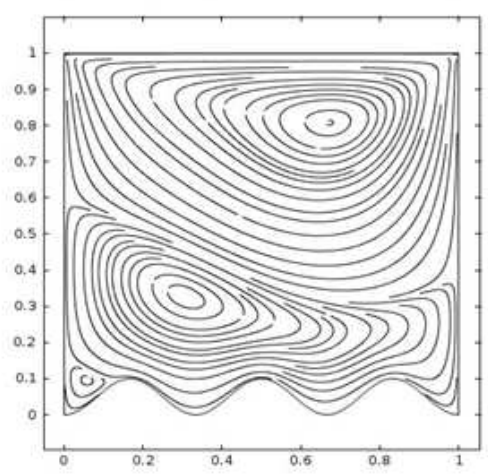

Fig 4. Stream line contour at $\operatorname{Re}=10^{2}, G r=10^{4}, A=0.05$ (a) $\lambda=0$ (b) $\lambda=1$ (c) $\lambda=2$ (d) $\lambda=3$.

(a)
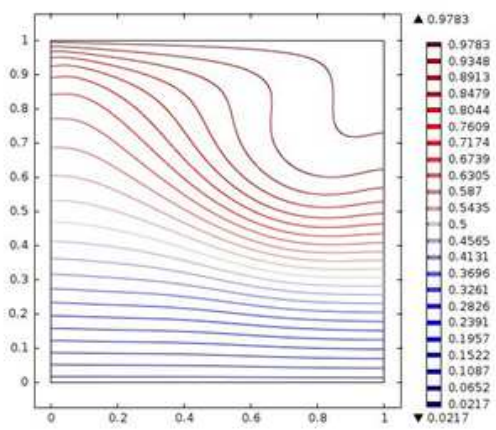

(c)

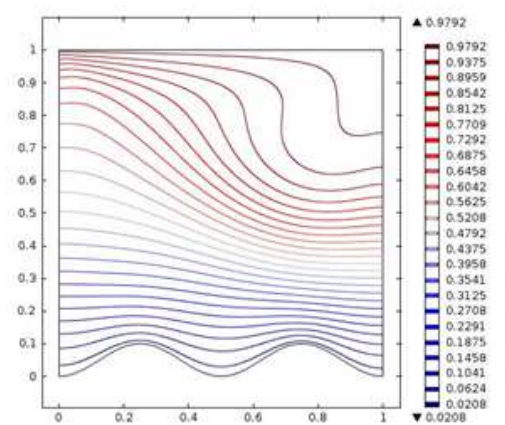

(b)

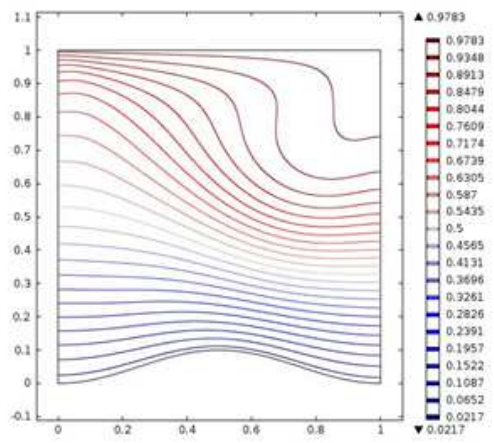

(d)

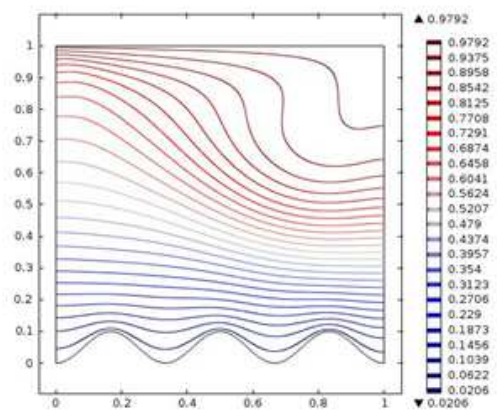

Fig 5. Isotherms contour at $\mathrm{Re}=10^{2}, G r=10^{4}, A=0.05$ (a) $\lambda=0$ (b) $\lambda=1$ (c) $\lambda=2$ (d) $\lambda=3$ 
The effect of varying Grashof number $\mathrm{Gr}$ on the streamlines contour and isotherms in a cavity for various undulations numbers with Reynolds number $R e=10^{2}$ is presented graphically in Figs 4-7. Here, the ratio of Grashof number and square of Reynolds number is unity, i.e., $\mathrm{Gr} / \mathrm{Re}^{2}$ which means Richardson number $\mathrm{Ri}=1$. So, according to the definition of fluid flow in the cavity the buoyancy effect balances the effect of moving upper wall and, hence the total heat transfer in the cavity lead by mixed convection which is the combined mechanism of forced convection and natural convection. When the buoyancy effect is comparable to the effect of heated sliding wall, the streamlines contour over the whole cavity region is occupied by two primary vortex as shown in Fig. 4. On the contrary, the conventional cavity flow with cooled sliding upper wall and heated lower wall the flow in the whole cavity region is occupied with a single primary vortex as discussed in [9]. It observes from the Figs. 4(b)-(d) that varying the number of undulations $\lambda$ from 1 to 3 does not affected the main cavity flow except a minor vortex that appears in the bottom left corner due to the wave from the bottom surface.

On the other hand, the effect of varying the number of undulation on the isotherms contour is shown in Figs. 5(a)-(d) at $\mathrm{Gr} / \mathrm{Re}^{2}=1$.It can be seen that the isotherms are clustered near the bottom surface which indicates that the temperature gradients are strong in this region. However, we see that the isotherms on the upper right corner of the cavity are weak which indicates that temperature gradients in this region are weak. It can be seen from the Figs. 5(a)-(d) that the isotherms do not affected by the variation of undulations numbers at the bottom surface except the vicinity of the

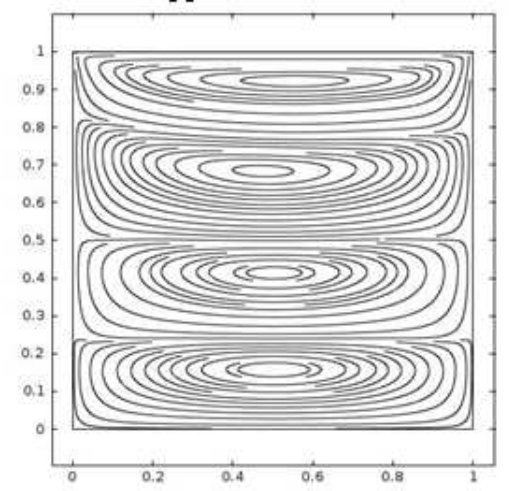

(c)

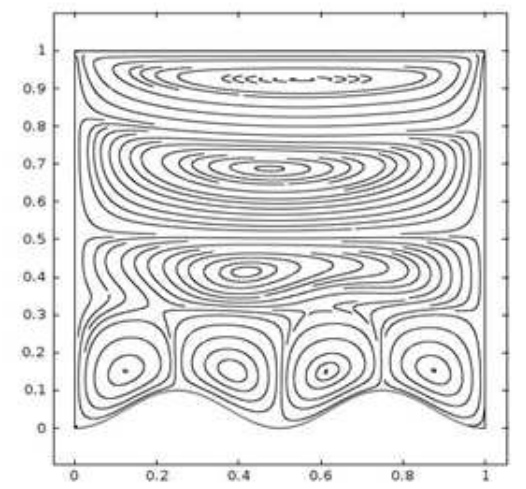

wavy bottom surface.

Next, the streamline contour and isotherms contour are illustrated in Figs 6-7 respectively for various undulations at Grashof number, $G r=10^{6}$. Here, the ratio of Grashof number and square of Reynolds number is 100 which means the Richardson number $R i=100$. So, according to the defininition of fluid flow in the whole cavity would be lead by free convection flow dominated by buoyancy force. Therefore, the impact of the mechanically drien top lid penetrates only a small distance towards the interior region. From Fig. 6(a) it is observed that the flow over the whole cavity is divided into four circular region when wavy surface is not employed at the bottom surface. It appears from Figs. 6(b)-(d) that empolyed the undulation numbers affect the distribution of steramlines contour in the vicinity of the wavy lower wall by dividing into two, four and six parts for one, two and three undulation numbers respectively. In this case flow lead by buoyancy force and increasing viscous effect produced by the wavy lower boundary, and hence, cavity flow in the vicinity of the lower boundary divided at the peaks of wavy surface depending on the number of undulations employed at bottom surface.

On the other hand, the influence of number of undulations on the isotherms contour is demonstrated in Figs 7 (a)-(d) with Richardson number, $\mathrm{Ri}=100$, i.e. the buoyancy effect outweighs the effect of sliding wall. This reflects the fact that heat transfer is mostly conductive except the upper portion of the cavity. The Figures show that varying the number of undulations does not change isotherm patterns except the vicinity of bottom wavy surface.

(b)

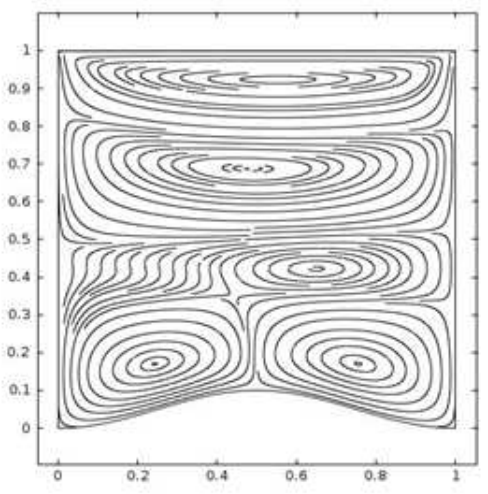

(d)

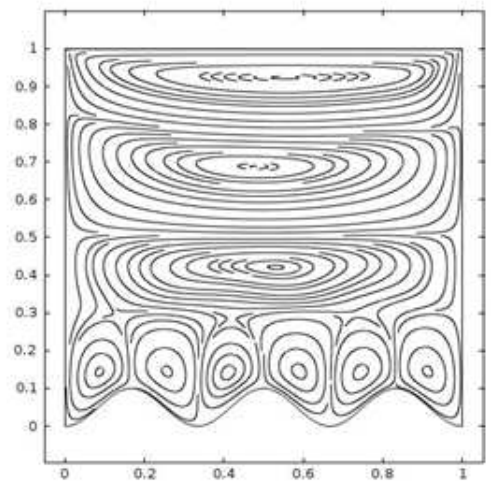

Fig 6. Stream line contour at $\mathrm{Re}=10^{2}, G r=10^{6}, A=0.05$ (a) $\lambda=0 \quad$ (b) $\lambda=1$ (c) $\lambda=2$ (d) $\lambda=3$. 
(a)

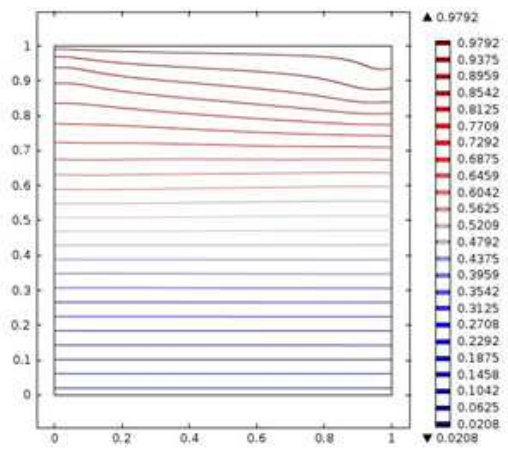

(c)

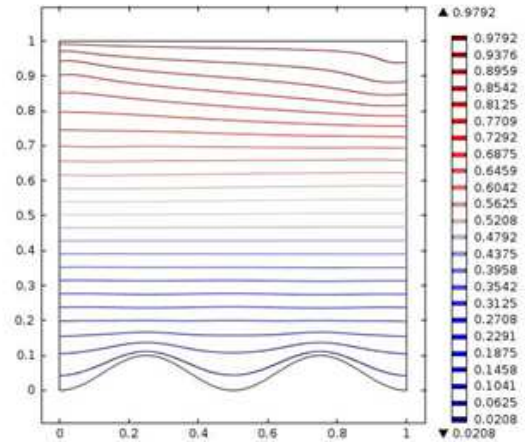

(b)

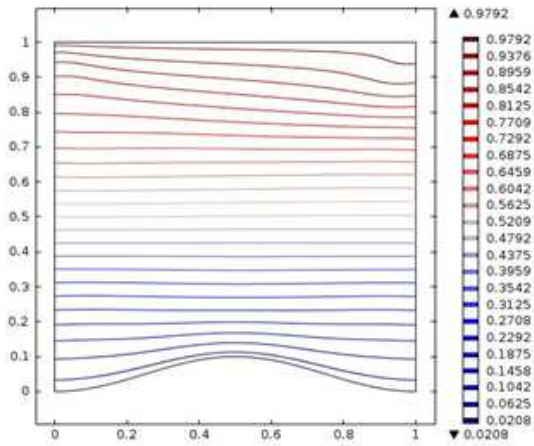

(d)

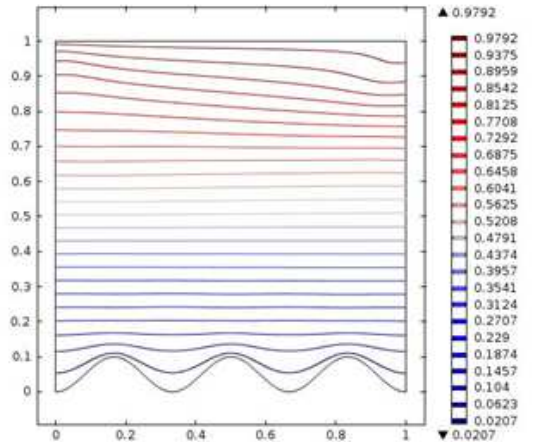

Fig 7. Isotherms contour at $\mathrm{Re}=10^{2}, G r=10^{6}, A=0.05$ (a) $\lambda=0 \quad$ (b) $\lambda=1$ (c) $\lambda=2$ (d) $\lambda=3$.

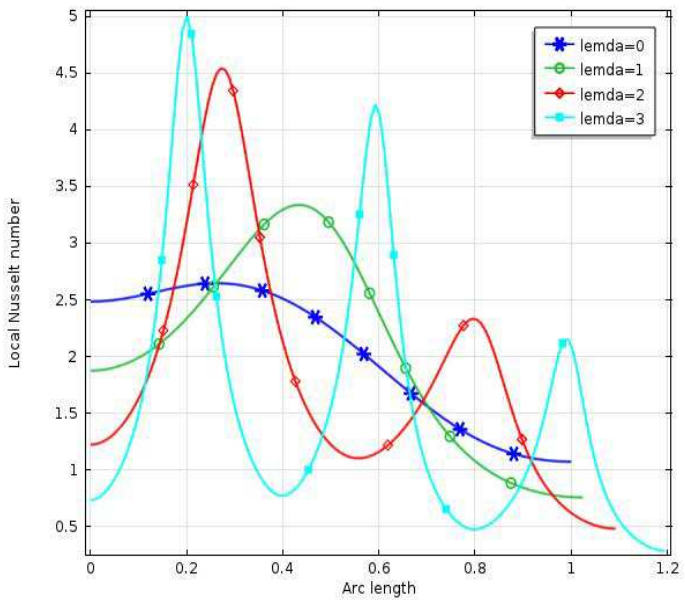

(a)

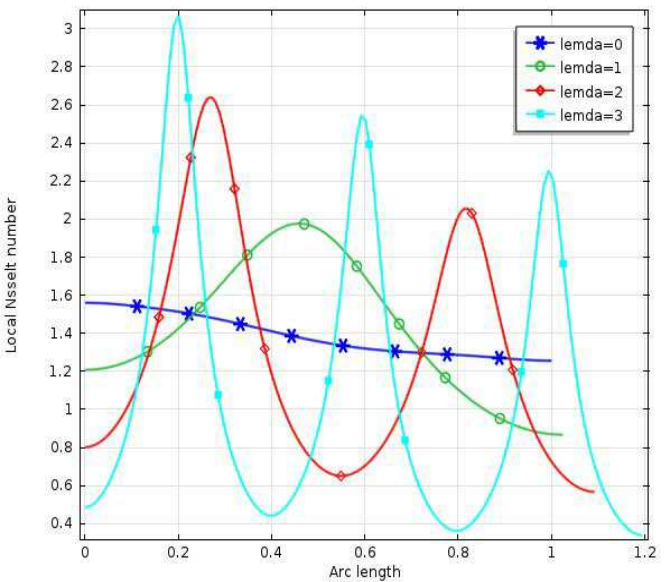

(b)

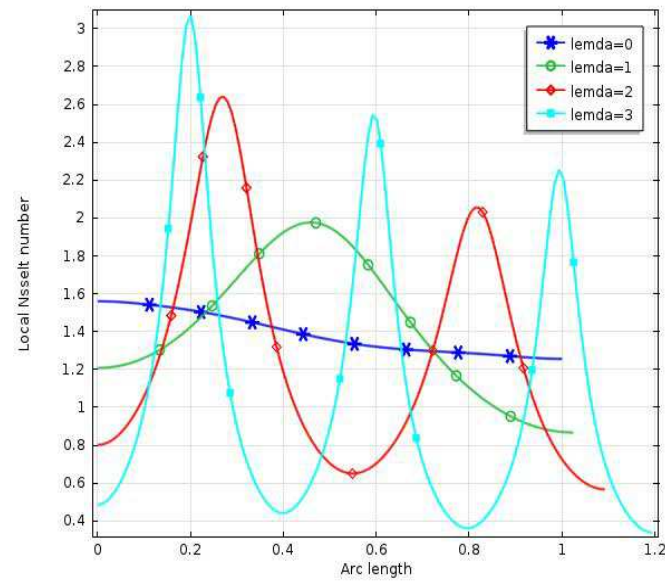

(c)

Fig 8. Local Nusselt numbers along the wavy bottom wall for various undulation, at the wavy bottom wall $A=0.05, \operatorname{Re}=10^{2}$ (a) $\mathrm{Gr}=10^{2}$ (b) $\mathrm{Gr}=10^{4}$ (c) $\mathrm{Gr}=10^{6}$

Figs. 8(a)-(c) display the local Nusselt numbers for various undulations with different Grashof numbers along the wavy bottom surface. The figure shows that the vertical temperature gradient along the arc length generally drops in the direction of lid motion due to the formation of thermal boundary layer over the surface. It appears that the employed number of undulation affects the distribution of the local heat flux along arc length of the bottom surface by producing a corresponding number of peaks and valleys, which correspond to the imposed $n$ values. It is worth pointing out that the highest local heat flux is achieved with $\mathrm{Gr} / \mathrm{Re}^{2}=\mathrm{Ri}=0.01$ which is likely attributed to the heat transfer augmentation offered by the mechanically sliding 
lid. It is also observed that increasing the values of Gr for a fixed $\mathrm{Re}, \mathrm{i}$. e. increasing values of $\mathrm{Ri}$ is found to decrease the highest peak for a given undulation number.

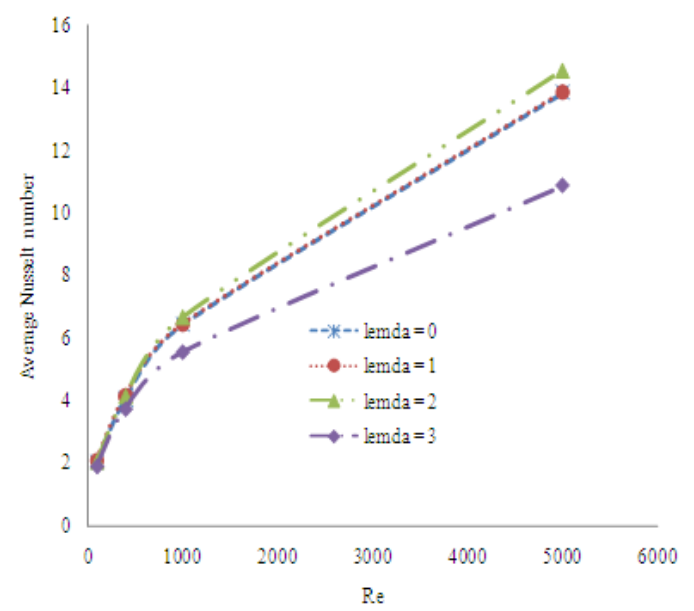

(a)

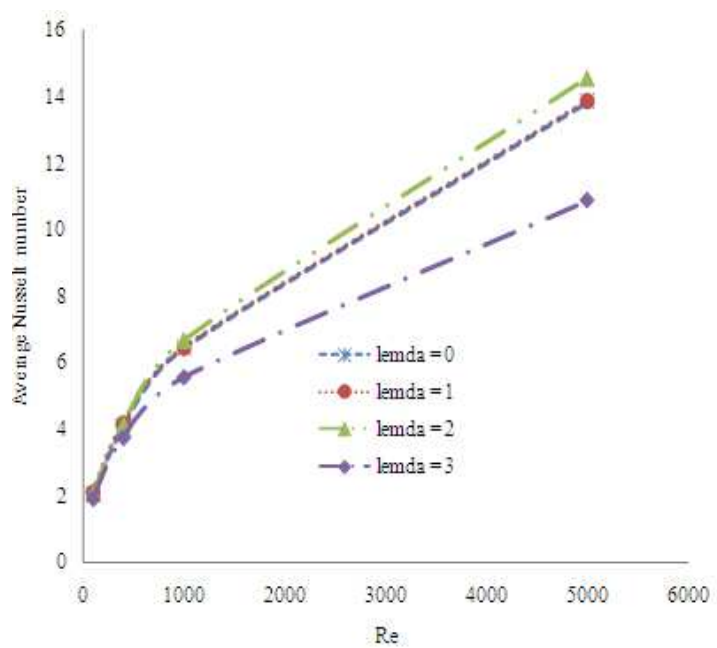

(b)

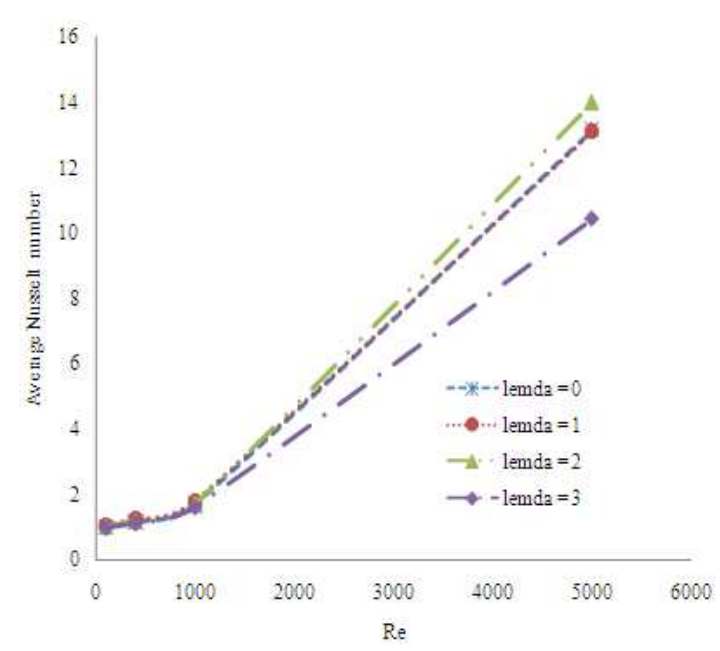

(c)

Fig 9. Variation of average Nusselt number along wavy bottom wall for various undulation, $A=0.05$ (a) $G r=10^{2}$ (b) $G r=10^{4}$ (c) $G r=10^{6}$.
The effect of the number of undulations on the average Nusselt number along wavy bottom surface for various Grashof number with various Reynolds numbers is presented in Fig. 9.

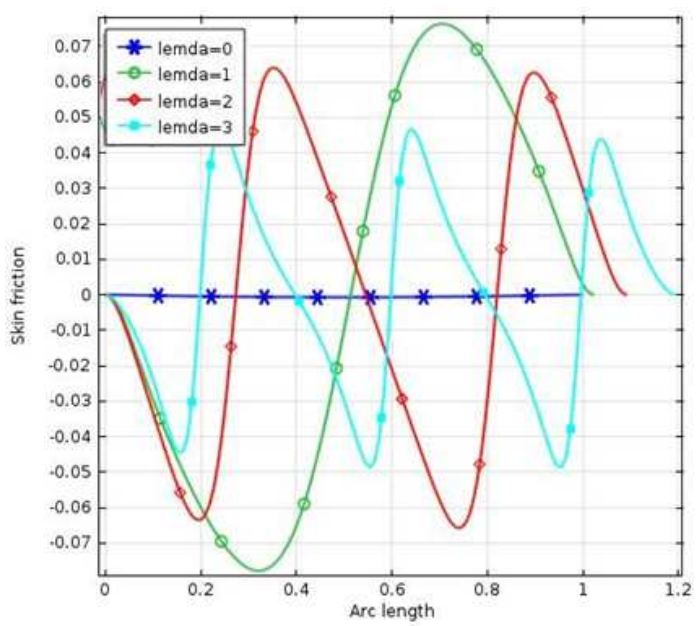

(a)

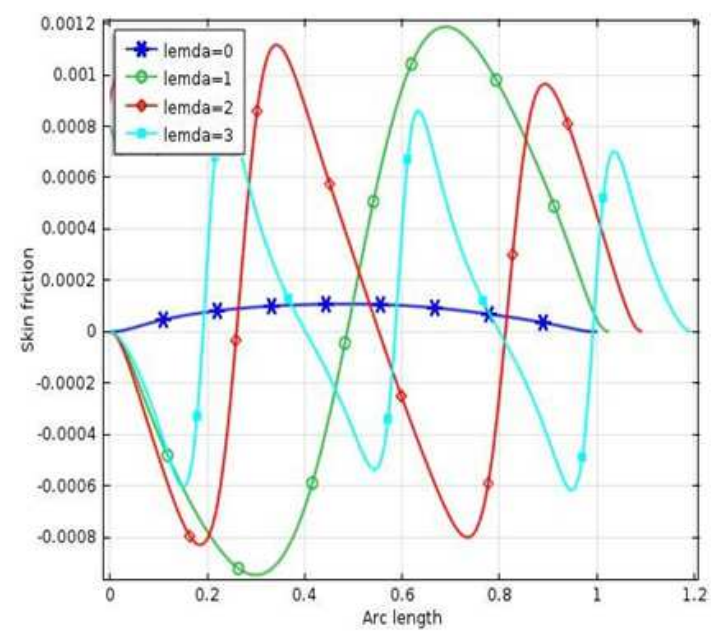

(b)

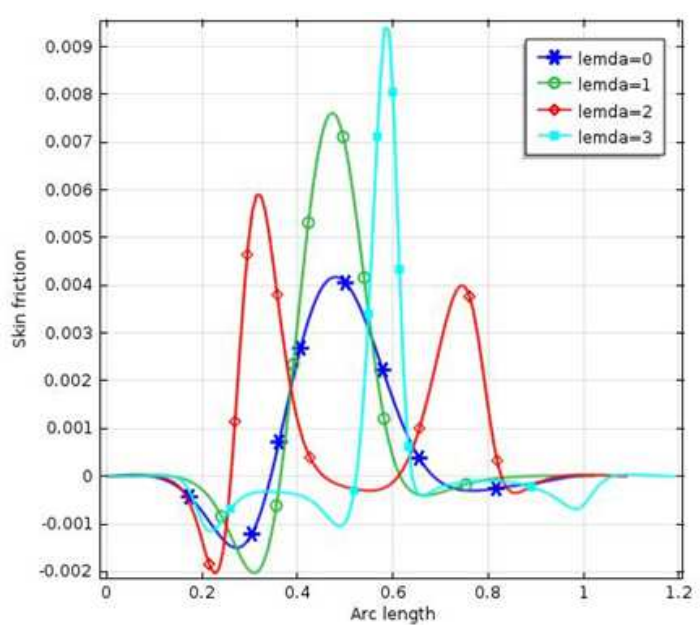

(c)

Fig 10. Variation of skin friction along bottom wavy wall for various undulations, $\mathrm{Gr}=10^{6}$ (a) $\operatorname{Re}=10^{2}$ (b) $\operatorname{Re}=10^{3}$ (c) $\operatorname{Re}=5 \times 10^{3}$ 


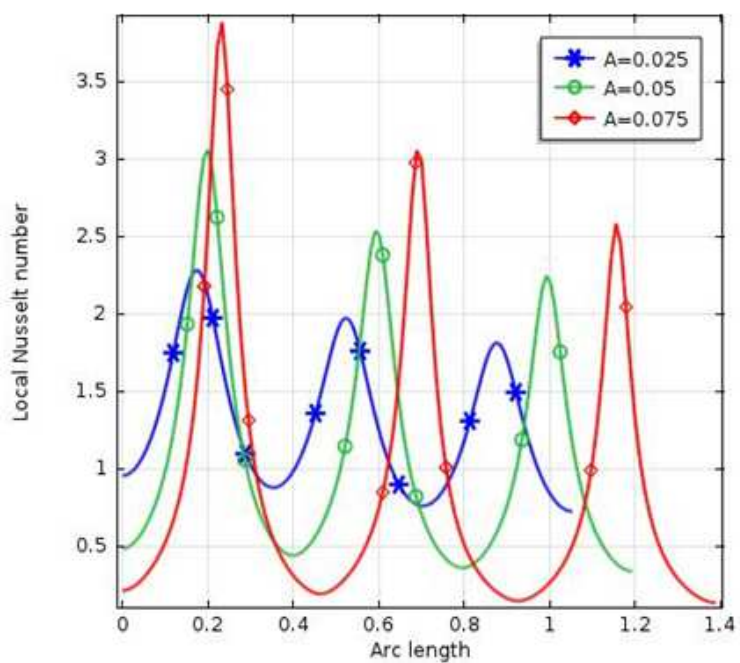

(a)

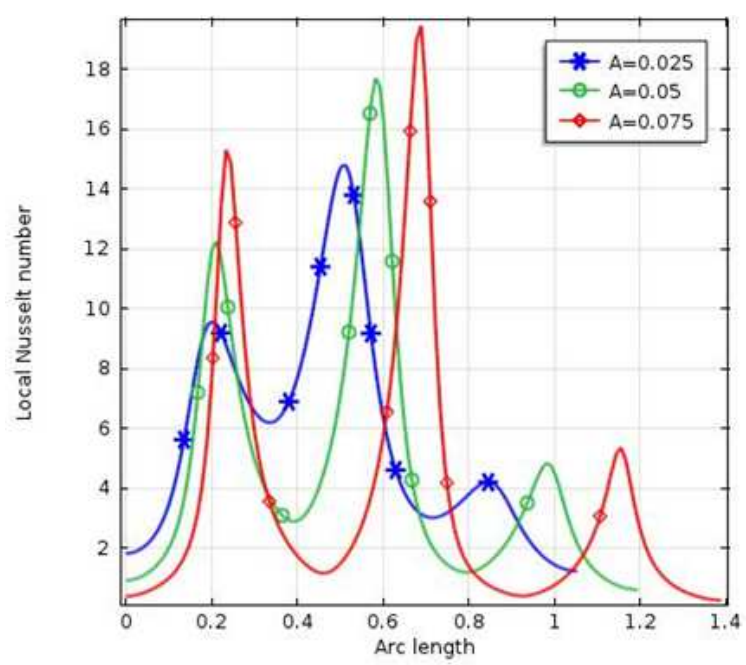

(b)

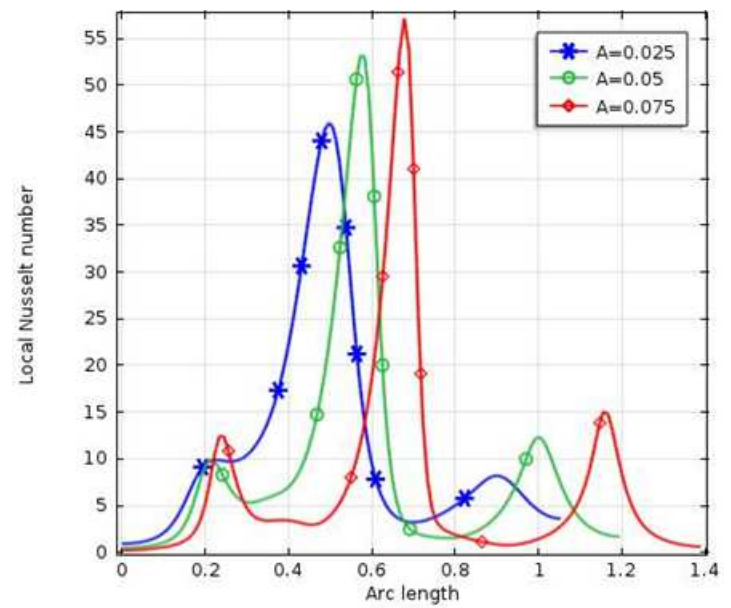

(c)

Fig 11. Local Nusselt number variation along wavy bottom line for various amplitude of the wavy surface, $\mathrm{Gr}=10^{6}$, (a) $\operatorname{Re}=10^{2}$ (b) $\mathrm{Re}=10^{3}$ (c) $R e=5 \times 10^{3}$.

From Fig. 9, it is observed that average nusselt number increases with the increasing values of Reynolds number for any given undulation number. Moreover, average Nusselt number profile remain same for $\lambda=0$ and $\lambda=1$, whereas the average Nusselt number peaks at $\lambda=2$ which reflects the high local Nusselt number as reported in Fig. 8. But, the average Nusselt number profile alter and less inflation occur when $\lambda=3$. It is observed that large value of average Nusselt number can be obtained by lower value of Grashof number, i.e. smaller value of Richardson number. On the other hand, average Nusselt number increases with the increase of Reynolds number, Re for any values of Grashof number, Gr.

The effect of Reynolds on the variation of skin friction along the wavy wall surface for various number of undulations are illustrated in Figs. 10(a)-(b). In this case, Grashof number is considered to be $\mathrm{Gr}=10^{6}$ which is sufficiently high. This implies that the buoyancy could accelerate the fluid traversing along the lid surface with the primary vortex. Therefore, the velocity of the fluid close to the lid surface could be faster than the moving speed of the lid. In that case, the skin friction may become negative. Recall the Fig. 6 we can see that there is no vortex at the bottom surface without employing the undulation number. As a result the skin friction is zero at the bottom surface for $\lambda=0$ at Reynolds number, $R e=10^{2}$. However, the skin friction is not zero for higher values of Reynolds number as the vortex may appear at bottom surface even the undulations not employed. When wavy bottom surface is considered the stream lines patterns characterized by a pair of counter rotating vortex (in Fig. 6). Therefore, the Fig. 10(a)-(b) represents the skin friction that shows the number of peaks and valleys which corresponds to the number of undulations imposed at bottom surface of the cavity. Hence, skin friction at bottom wall simply follows the streamlines contour. Meanwhile, for high values of Reynolds number i.e. $\operatorname{Re}=5 \times 10^{3}$ the influence of lower minor vortices becomes so stronger that it suppressed the cavity flow of the lower bottom surface. As a result, the effect of undulation number on the skin friction does not follow the same tendency as discussed in Fig 10(a) and (b).

Fig.11 shows the effect of the amplitude on the local variation of Nusselt number along wavy bottom surface of the cavity. The results in Fig. 11 demonstrates higher local Nusselt number with an increase in the amplitude of the wavy surface due to the increase of velocity gradients near the top lid which subsequently increase the rate of heat transfer. It is also observed that the inflation of the amplitude of the wave increase the rate of heat transfer (local Nusselt number) in the concave up region and it becomes highest at the upper peak point. On the other hand, the rate of heat transfer decreases in the concave down region and it becomes lowest at the lower peak point. Moreover, the heat flux along the wavy surface increases with the increase of Reynolds number, Re.

\section{Conclusion}

This paper presents a numerical study to examine the effects of wavy bottom surface on the mixed convection heat 
transfer in a lid-driven cavity heated from top for various pertinent dimensionless groups. Insulated boundary conditions are imposed to vertical side walls. The governing equations are solved using the Galerkin finite element method. Comparisons with previously published work on special cases of the problem were made and found to be in good agreement. Graphical results for various parametric conditions were presented and discussed. It was found that the heat transfer mechanisms and the flow characteristics inside the cavity are strongly dependent on the number of undulations, Grashof number and Reynolds number. When the ratio of Grashof number and square of Reynolds number (Richardson number) is greater or equal to unity then the streamline contours over the whole cavity region is occupied by several primary vortex. In this case, heat transfer is mainly through conduction except the upper portion of the cavity. The isotherms are not affected by the variation of undulation numbers at the bottom surface except the vicinity of the wavy bottom surface. The skin friction and local Nusselt number increase with increasing values of Reynolds number for any values of undulation number. Finally, the prediction of the local and average Nusselt number is found to increase with larger values of Grashof number and higher amplitude of the wavy surface. Thus, the wavy lid-driven cavity can be considered as an effective heat transfer mechanism at larger wavy surface amplitudes and higher Grashof number.

\section{References}

[1] C. K. Cha, Y. Jaluria, "Recirculating mixed convection flow for energy extraction”, Int. J. Heat Mass Transfer, (1984), 27, pp-1801-1810.

[2] L.A.B. Pilkington, "Review lecture, The Float Glass Process", third edition: Proc. R. Soc. Lon., (1969), 314, pp-1-25.
[3] J. Imberger, P.F. Hamblin, "Dynamics of lakes, reservoirs, and cooling ponds", Adv. Rev. Fluid Mech, (1982), 14, pp-153-187.

[4] A.K. Prasad, J.R. Koseff, Combined forced and natural convection heat transfer in a deep lid-driven cavity flow, Int. J. Heat Fluid Flow, (1996), 17, pp 460-467.

[5] P.K. Das, S. Mahmud, "Numerical investigation of natural convection inside a wavy enclosure", Int. J. Therm. Sci, (2003), 42, pp-397-406.

[6] L. Adjlout, O. Imine, A. Azzi, M. Belkadi, "Laminar natural convection in an inclined cavity with a wavy-wall", Int. J. Heat Mass Transfer, (2002), 45, pp-2141-2152.

[7] B.V.R. Kumar, "A study of free convection induced by a vertical wavy surface with heat flux in a porous enclosure", Num. Heat Transfer,Part A (2000), 37, pp-493-510.

[8] K. Khanafer, B. Al-Azmi, Alia Marafie, I. Pop, Non-Darcian effects on natural convection heat transfer in a wavy porous enclosure, Int. J. Heat Mass Transfer, (2009), 52, pp. $1887-1896$

[9] A. M. Al-Amiri, K.M. Khanafer, I. Pop, Effect of sinusoidal wavy bottom surface on mixed convection heat transfer in a lid driven cavity, Int. J. Heat Mass Transfer, (2007), 50, pp. $1771-1780$

[10] C. Taylor, P. Hood, A numerical solution of the Navier-Stokes equations using finite element technique, Computers \& Fluids 1 (1) (1973) 73-89.

[11] P. Dechaumphai, Finite Element Method in Engineering, 2nd ed. Chulalongkorn University Press, Bangkok, 1999.

[12] R. Iwatsu, J. M. Hyun, K. Kuwahara, "Mixed convection in a driven cavity with a stable vertical temperature gradient", Int. J. Heat Mass Trsmfer, (1993), 36(6), pp-1601-1608.

[13] M. A. H. Mamun, T. R. Tanim, M. M. Rahman, R. Saidur and Shuichi Nagata, "Analysis of Mixed Convection in a Lid Driven Trapezoidal Cavity". 